\title{
Role of Maxillofacial Radiologist in Ballistic Wound: Case Report with Literature Review
}

\author{
Nishat Sultana, Ehtaih Sham \\ Vydehi Institute of Medical \& Dental Sciences, Bangalore, India \\ Email: nishat_tamanna9@rediffmail.com, ehtaihsham@yahoo.com
}

Received May 4, 2012; revised June 8, 2012; accepted July 1, 2012

\begin{abstract}
Gunshot injuries are rather serious but uncommon type of trauma in India. Radiologists can contribute substantially in evaluation and treatment of patients with a gunshot wounds. Plain films, CT, Angiography, and sometimes MR imaging are used to localize shots. This paper describes a case report of shotgun injury to the face and neck and also attempts to illustrate the spectrum of available imaging with relevant findings pertaining to bullets and shotgun pellets in gunshot injuries. Radiologists should be aware of the associated complications and forensic implications when they take on the task of interpreting these images.
\end{abstract}

Keywords: Ballistic Wound; Maxillofacial Radiologist; Projectile

\section{Introduction}

Any discussion of gunshot injury tends to evoke emotional reaction among citizens. Firearm-related injuries are the second leading cause of injury-related deaths in United States [1]. In India incidence of firearm homicide rate is about $0.93 \%$ according to the United Nations office on drugs and crime 2000 [2]. 14\% of all gunshot-related assaults will result in maxillofacial injuries [1]. Although there has been an increase in the incidence of gunshot wounds to the face [3], gunshot-related craniofacial injuries are still not as common as those to other regions of the body $[4,5]$.

If this trend continues, then the mortality rate related to fire arms would soon exceed motor vehicle accidents, which are more common cause of death secondary to maxillofacial/head injury [4]. The role of radiologist in forensic investigation classically has been to asset in identification of human remains, but the informed radiologist can contribute much more, particularly in forensic investigation of fatal gunshot wounds. Any radiologist might be asked to assist in a forensic investigation involving firearms, and it is Important that the radiologist be aware of what information can and cannot be obtained from a radiograph. In this article we attempt to present importance of radiologist and radiology in evaluating bullet injuries.

\section{Case Report}

A 40-year-old male patient reported to an emergency department with gunshot injury on left side of his neck from a close range. Patient was unconscious but was sta- ble; with all vitals within normal limits. On examination postero-lateral part of neck on left side and left lower half of face had multiple small entry wounds each surrounded by a grayish to black color halo with serous type of discharge, the surrounding area was lacerated and skin over the face and neck was inflamed (Figure 1). Routine hematology and radiological investigations were carried out and blood transfusion given to compensate blood loss. Reconstructed 3D CT (Figure 2), PA mandible with $\mathrm{C}$-spine was taken (Figure 3). This showed multiple splinters/pellets from shot gun present on the left postero-lateral neck and front and lower half of face with comminuted fracture of the angle/lower border of mandible due to the impact from gun splinters. One of the splinter was lodged in inter vertebral space between $\mathrm{C} 4$ and C5 (Figure 4) which lead to neurological deficit. Surgical exploration \& debridement of the site was done with removal of few splinters/pellets (Figure 5) and with repair of fracture angle/lower border of mandible (Figure 6), splinter/pellet at the intervertebral space was not removed at the time of first surgery as it could cause further damage \&increase the neurologic deficit. Wound closed primarily (Figure 7). Patient was routinely evaluated in order to access whether there was an increase or decrease in his neurologic status. Patient has been recalled again for a second surgical procedure for removal of splinter from intervertebral space (Figure 8).

\section{Discussion}

Bullet injuries are divided into high-velocity $\left(>2000 \mathrm{ft} \cdot \mathrm{s}^{-1}\right)$ and low-velocity $\left(<2000 \mathrm{ft} \cdot \mathrm{s}^{-1}\right)$ [6]. A high velocity 


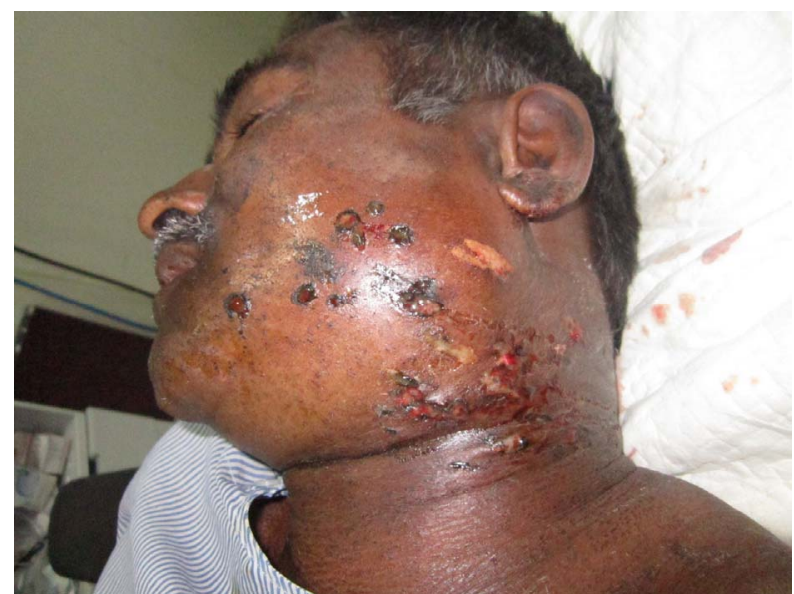

Figure 1. Multiple small entry wounds.

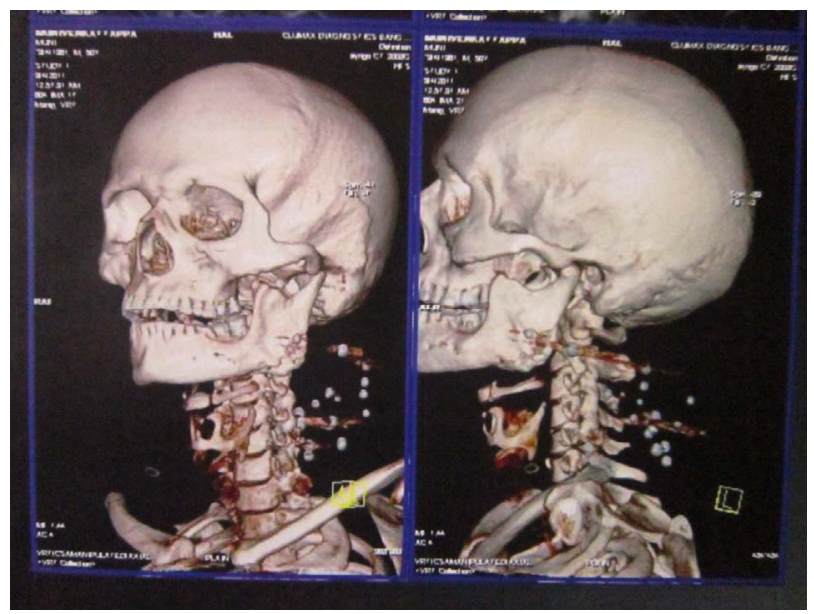

Figure 2. Reconstructed 3D CT shows multiple pellets on the left postero-lateral neck and front and lower half of face.

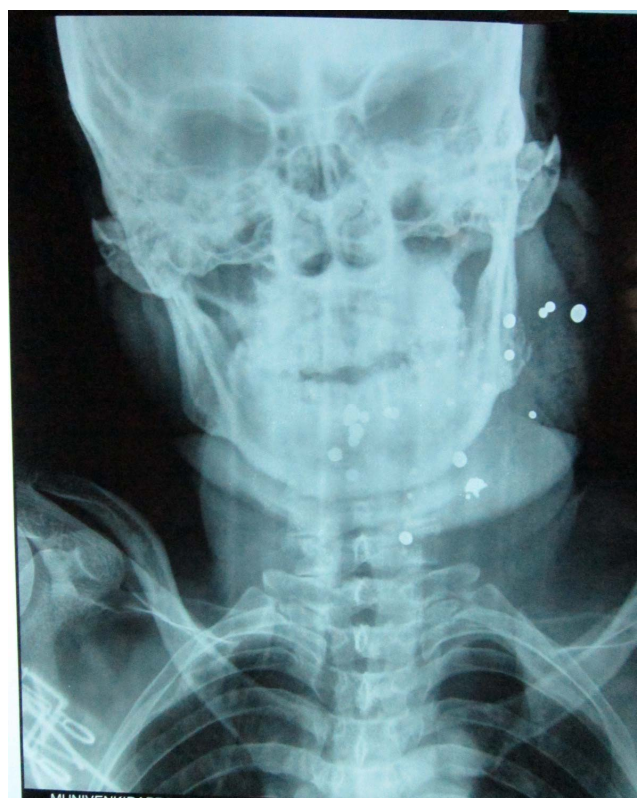

Figure 3. PA mandible with C-spine.

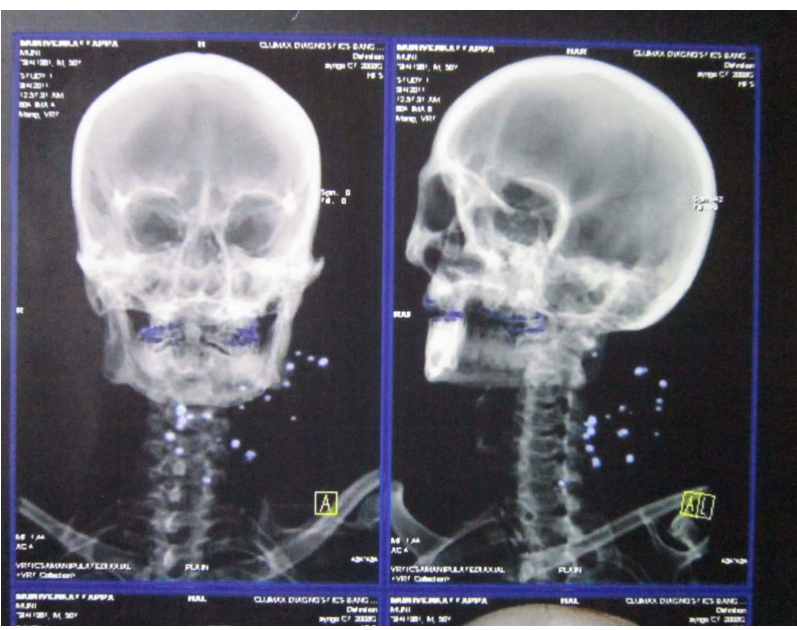

Figure 4. Splinter was lodged in inter vertebral space between $\mathrm{C} 4$ and $\mathrm{C5}$.

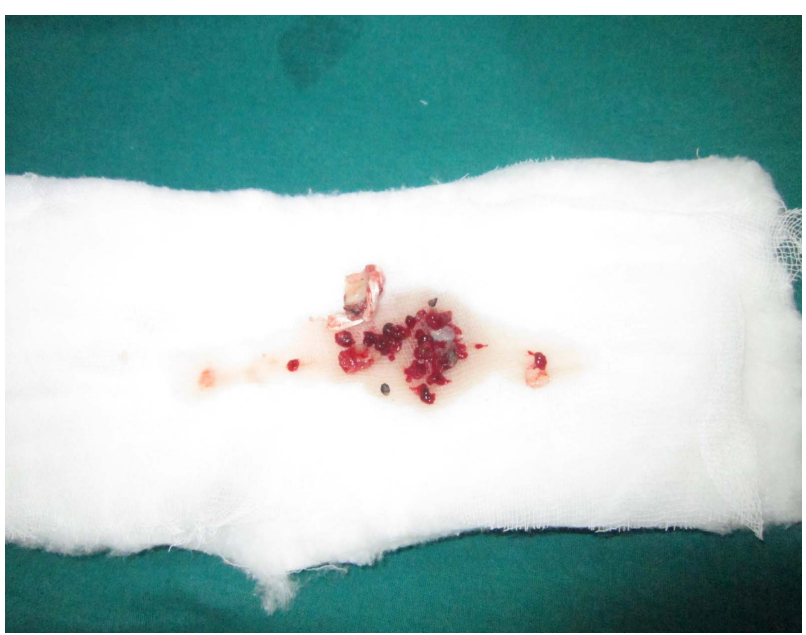

Figure 5. Surgical exploration \& debridement of the site was done with removal of few splinters/pellets.

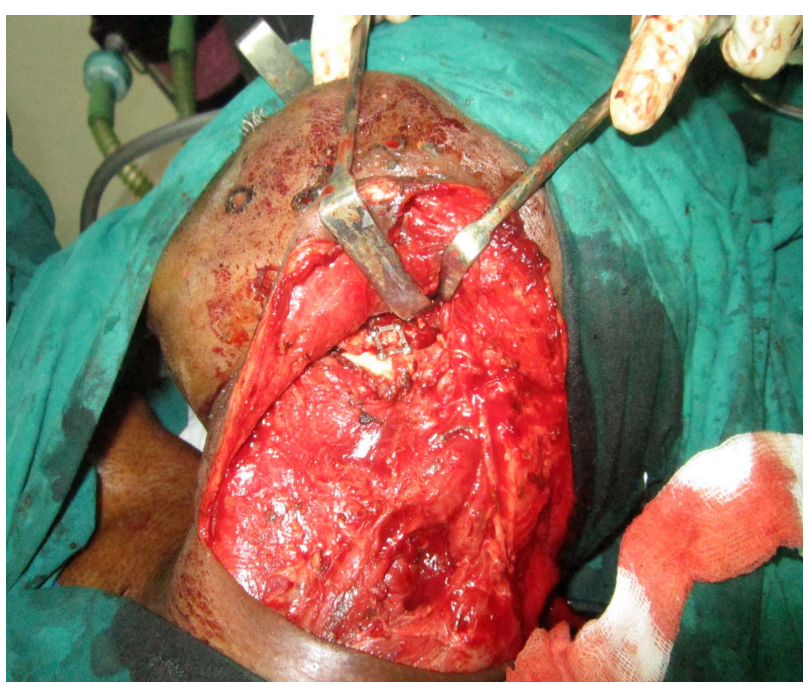

Figure 6. Repair of fracture angle/lower border of mandible. 


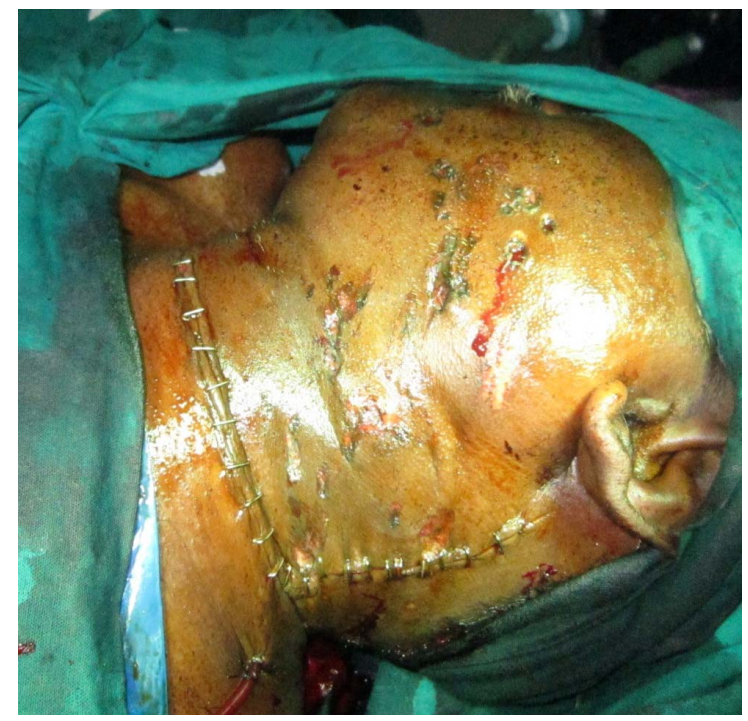

Figure 7. Wound closed primarily.

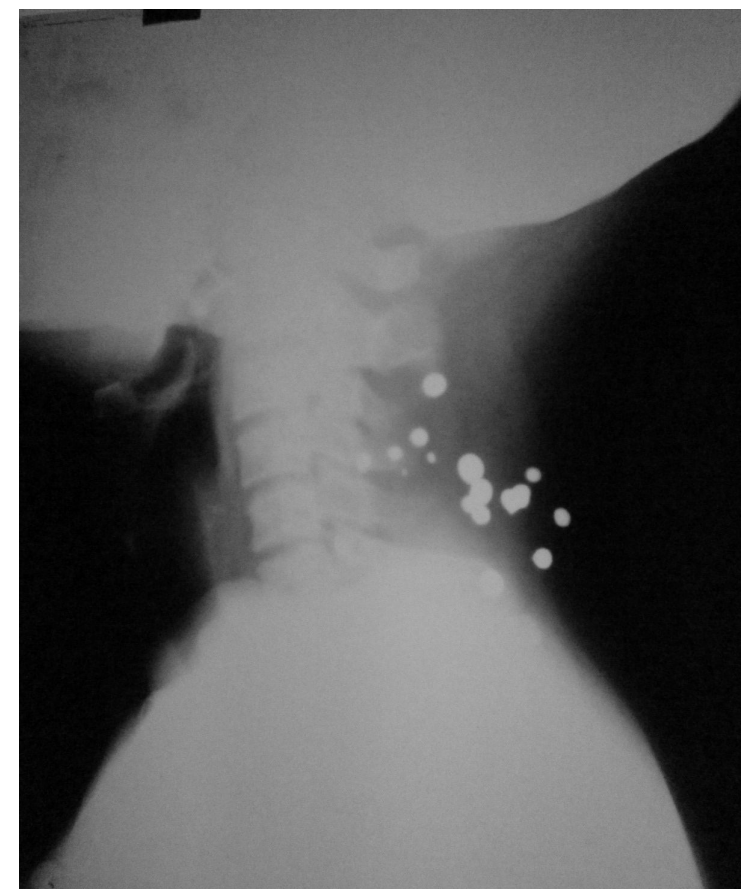

Figure 8. Post operative radiograph.

bullet is likely to lead to quick and fatal injury to the victim, whereas a low-velocity bullet may result in a nonfatal injury. It is, therefore, likely that the oral maxillofacial radiologist may encounter both low $\&$ high velocity bullet injuries to the maxillofacial region [7].

\section{Mechanism of Gunshot Injury}

Bullet injuries are most severe in friable solid organs (e.g. liver and brain) where damage may be caused by temporary cavitations (tissue stretch) remote from the actual bullet track [8]. Dense tissue (e.g. bone) and loose tissue (e.g. subcutaneous fat) are more resistant to bullet injury. Bone modifies the behavior of bullets markedly, by altering their course, creating a tumbling effect, slowing them down and increasing deformity and fragmentation $[8,9]$. Evaluation of bone injuries and the distribution of bone and bullet fragments on radiographs can be helpful in determining the direction of travel, which is important not only for clinical assessment but also for forensic evaluation of the incident [10].

The degree of bullet fragmentation is also affected by bullet construction. The presence of a full or partial metal jacket has a major effect on deformity. Bullets with full metal jackets often remain in one piece and usually do not deform much. These projectiles typically do not leave a trial of lead fragments along their path. On the other hand semi jacketed, hollow point, non jacketed soft point bullets tend to deform on impact or break apart, leaving a telltale trail of metal fragments through the soft tissue [11].

As the projectile enters the victim, the different layer of tissue reacts accordingly to their specific properties. Injuries to the dermis include abrasion, impaction of particulate matter, and contusion. At closer ranges, burning and implantation of powder and residue may occur and may result in a tattoo. After the projectile passes through the skin, it next encounters muscle tissue, which is very elastic and may sustain deformation of as much as four times the diameter of the projectile. The shape of this deformation will be similar to that of the temporary cavity. On a cellular level, the muscle along the pathway of the projectile becomes devitalized and necrotic. As the projectile travels, it may also encounter other vital structures such as nerves and blood vessels. The injuries to neurovascular tissue are similar to injuries to muscle. Vessels may be ruptured, crushed, or sheared, and spasm may occur. These injuries may result in hemorrhage and in the formation of thrombi and hematoma. On a cellular level, damage occurs to all three layers of the vessel wall [12]. Injury to bony tissue differs from injury to soft tissues. The minimal projectile velocity required for bone fracture is $65 \mathrm{~m} / \mathrm{s}$. Bone is basically inelastic; therefore, the type of injury that occurs depends on the type of bone encountered by the projectile. Injury to cancellous bone usually results in a defect of the drill-hole type. Injury to cortical bone or teeth usually results in shattering. The resulting fragments may act as secondary projectiles and may pose an aspiration risk [13].

Glazer and collegues [14] divided the shot gun injuries to three types focusing on the surface area of pellet scattered.

Type I: Injuries result when scattered is contained within an area of $25 \mathrm{~cm}^{2}$ and the pellets act as individual missiles.

Type II: Injuries were defined as pellet scatter contained within an area of $10 \mathrm{~cm}^{2}$ to $25 \mathrm{~cm}^{2}$. 
Type III: Injuries result from scattered contained within area of less than $10 \mathrm{~cm}^{2}$.

Radiographic appearance of bullet injuries:

Fragmentation of high velocity bullet creates a lead snowstorm appearance on radiographs $[8,11]$. The area over which the lead snowstorm fragments are deposited in the soft tissues widens as the distance from the entry site increases. Thus, a conical distribution of lead fragments is seen on radiograph with the apex of the cone pointing towards the entry side [8].

CT and 3DCT is the best in demonstrating the location of the object, cavitations and metal and bone fragment created by high velocity missiles but less accurate for detecting wood, clothing plastics, stones, and other relatively radiolucent material propelled into the wound by the bullet [15]. MR imaging can be useful in the evaluation of gunshot wounds, particularly when star artifact from a dense metallic bullet fragment limits the usefulness of CT [16]. Vascular abnormalities, including arteriovenous fistula or aneurysm, sometimes are revealed [17]. But in case of shot guns where pellet (shots) were made up of steel, they are ferromagnetic and can move if the patients is exposed to strong magnetic field, thus causing additional damage. Fortunately steel and lead pellets can usually be distinguished from one another at radiography. Lead pellets tend to be deformed and fragmented by impact with soft tissue and bone. Simple analysis of a radiograph is all that is needed to determine if a patient with shot gun injury can be safely placed in MR imaging magnet [9].

Color flow Doppler and CT angiography are the radiological investigation tools of the head and neck injuries that have the potential of involving great vessels in the region $[18,19]$. On gray-scale sonographic images it is possible to localize bullets relative to blood vessels because metal is hyper echoic and produces a characteristic trailing band of increased echogenicity (posterior reverberation or "comet tail") [20].

Cone beam CT (CBCT) scans may be an important tool as this would lead to fewer artifacts. However, it needs to be noted that with the limited field of view offered by CBCT the extent of damage caused by a bullet injury could never be completely assessed. Moreover, in developing countries, such as ours, $\mathrm{CBCT}$ facilities are scarce and expensive [7].

Radiopaque marker used with 3DCT reconstruction placed over entry and exit wound have been used to help and evaluate penetrating injuries and provide a permanent record of wound location and assess the damage to vital structure [21].

\section{Conclusions}

In conclusion "the only rule regarding the science of ballistics is that the bullet follows no rule" by Hough [22].
Not all gunshot wounds are same. Gunshot injuries will vary depending on the type of bullet used, the distance from which the bullet was fired, type of shot (size and weight of pellets), impact velocity and body tissue resistance.

A radiologist who is familiar with the basic principles of wound ballistics and who is available when the patient arrives can have a major effect on imaging and management, prompt and accurate assessment of the injuries is essential both clinically and radiographically.

In our case 3DCT and C-spine PA view was done which accurately determined the position of shots. Though all the shots were not removed, the clinical benefits of pellet removal surpass then possible surgical post operative complications, with the improvement in neurological symptoms, we opted for wait and watch policy with periodic follow ups with consent of the patient.

\section{REFERENCES}

[1] J. L. Amnest, J. A. Mercy and G. W. Ryan, "Surveillance Gotsch Fatal and Nonfatal of Firearms-Related Injuries-United States, 1993-1998," MMWR Morb Mortal Wkly Rep., 2001.

[2] "The Seventh United Nations Survey on Crime Trends and the Operations of Criminal Justice Systems (19982000)," United Nations Office on Drugs and Crime (UNODC).

[3] D. Demetriades, S. Chahwan, H. Gomez, A. Falabella, G. Velmahos and D. Yamashita, "Initial Evaluation and Management of Gunshot Wounds to the Face," Journal of Trauma-Injury Infection \& Critical Care, Vol. 45, No. 1, 1998, pp. 39-41. doi:10.1097/00005373-199807000-00007

[4] D. Puzovic, V. S Konstatinovic and M. Dimitrijevic, "Evaluation of Maxillofacial Weapon Injury: 15-Year Experience in Belgrade," Journal of Craniofacial Surgery, Vol. 15 , No. 4, 2004, pp. 543-546. doi:10.1097/00001665-200407000-00003

[5] A. Cowey, P. Mitchell, I. Gregory, I. Maclennen and R. Pearson, "A Review of 187 Gunshot Wound Admissions to a Teaching Hospital over 54-Month Period: Training and Service Implications," Annals of the Royal College of Surgeons of England, Vol. 86, No. 2, 2004, pp. 104-107. doi: $10.1308 / 003588404322827482$

[6] L. Cunninghum, R. Hough and J. Ford, "Firearm Injuries to the Maxillofacial Region: An Overview of Correct Thoughts Regarding Demographics, Pathophysiology and Management," Journal of Oral and Maxillofacial Surgery, Vol. 65, No. 8, 2003, pp. 932-942. doi:10.1016/S0278-2391(03)00293-3

[7] K. Sansare, V. Khanna and F. Karjodkar, "The Role of Maxillofacial Radiologists in Gunshot Injuries: A Hypothesized Missile Trajectory in Two Case Reports," Dentomaxillofacial Radiology, Vol. 40, No. 1, 2011, pp. 53-59. doi:10.1259/dmfr/72527764

[8] A. J. Wilson, "Gunshot Injuries: What Does a Radiologist Need to Know? Imaging Symposium," Radio Graphics, 
Vol. 19, No. 5, 1999, pp. 1359-1368.

[9] K. G. Swan and R. C. Swan, "Principles of Ballistics Applicable to the Treatment of Gunshot Wounds," The Surgical Clinics of North America, Vol. 71, No. 2, 1991, pp. 221-239.

[10] C. H. Choi, J. Pritchard and J. Richard, "Path of Bullet and Injuries Determined by Radiography," American Journal of Forensic Medicine \& Pathology, Vol. 11, No. 3, 1990, pp. 240-245.

[11] C. D. Phillips, "Emergent Radiologic Evaluation of the Gunshot Wound Victim," The Radiologic Clinics of North America, Vol. 30, No. 2, 1992, pp. 307-324.

[12] Y. H. Tan, S. X. Zhou and Y. Q. Liu, "Small-Vessel Pathology and Anastomosis Following Maxillofacial Firearm Wounds: An Experimental Study," Journal of Oral and Maxillofacial Surgery, Vol. 49, No. 4, 1991, pp. 348352. doi:10.1016/0278-2391(91)90368-V

[13] M. Oehmichen, C. Meissner and H. G. König, "Brain Injury after Gunshot Wounding: Morphometric Analysis of Cell Destruction Caused by Temporary Cavitation," Journal of Neurotrauma, Vol. 17, No. 2, 2000, pp. 155-162. doi:10.1089/neu.2000.17.155

[14] J. A. Glezer, G. Minard, M. A. Croce, T. C. Fabian and K. A. Kudsk, "Shotgun Wounds to the Abdomen," The American Surgeon, Vol. 59, No. 2, 1993, pp. 129-132.

[15] M. Can, N. Yildirin and K. G. Atore, "Dissecting Firearm Injury to the Head and Neck with Non-Linear Bullet Trajectory: A Case Report," Forensic Science International, Vol. 197, No. 1, 2010, pp. e13-e17. doi:10.1016/j.forsciint.2009.12.050

[16] N. A. Ebraheim, E. R. Savolaine, W. T. Jackson, T. G. Andreshak and A. Aayport, "Magnetic Resonance Imag- ing in the Evaluation of a Gunshot Wound to the Cervical Spine," Journal of Orthopaedic Trauma, Vol. 3, No. 1, 1989, pp. 19-22. doi:10.1097/00005131-198903010-00004

[17] W. C. Hanigan, A. M. Wright, W. A. Berkman and T. E. Szymke, "MR Imaging of a False Carotid Aneurysm," Stroke, Vol. 17, No. 6, 1986, pp. 1317-1319. doi:10.1161/01.STR.17.6.1317

[18] C. J. Fox, D. L. Gillespie, M. A. Weber, et al., "Delayed Evaluation of Combat-Related Penetrating Neck Trauma," Journal of Vascular Surgery, Vol. 44, No. 1, 2006, pp. 86-93. doi:10.1016/j.jvs.2006.02.058

[19] D. Demetriades, D. Theodorou, E. Cornwell, et al., "Penetrating Injuries of the Neck in Stable Patients: Physical Examination, Angiography or Colour Flow Doppler Imaging," Archives of Surgery, Vol. 130, No. 9, 1995, pp. 971-975. doi:10.1001/archsurg.1995.01430090057019

[20] T. R. Bonk, D. S. Harisson and H. M. Meissner, "Intravascular Bullet Localization by Sonography," American Journal of Roentgenology, Vol. 167, No. 1, 1996, pp. 151-152.

[21] A. Ramasamy, D. E. Hinsley and A. J. Brooks, "The Use of Improvised Bullet Markers with 3D CT Reconstruction in the Evaluation of Penetrating Trauma," Journal of the Royal Army Medical Corps, Vol. 154, No. 4, 2008, pp. 239-241.

[22] R. H. Hough, "Gunshot Wounds to the Head and Neck," In: J. P. W. Kelly, Ed., Oral and Maxillofacial Surgery. Knowledge Update, American Association of Oral and Maxillofacial Surgeons, Chicago, 1995, pp. 65-68. 